Olivia Mitscherlich-Schönherr

\title{
Editorial: Das Gelingen der Künstlichen Natürlichkeit
}

\author{
Mensch-Sein an den Grenzen des Lebens unter den \\ Bedingungen disruptiver Biotechnologien
}

\section{Zu den historischen Kontexten des Bandes}

Die Aufsätze, die der vorliegende Band versammelt, sind im Laufe des Jahres 2020 bzw. im Frühjahr des Jahres 2021 entstanden. Sie stammen damit aus einer Zeit, die von der globalen Corona-Krise und den politischen Maßnahmen und soziokulturellen Praktiken ihrer Bewältigung bestimmt war.

Vor dem Hintergrund dieser Entwicklungen geht der Band Fragen nach dem Mensch-Sein unter den Bedingungen disruptiver Biotechnologien nach. Die disruptiven Biotechnologien, die sich der vorliegende Band zum Gegenstand macht, gehören zu den sogenannten Konvergenz- bzw. NBIC-Wissenschaften und -Technologien: den Nano-, Bio-, Info- und Kognitionswissenschaften incl. Neurowissenschaften und Hirnforschung (vgl. Coenen 2008, 107). Im Zentrum der Konvergenzen zwischen diesen Wissenschaften und Technologien stehen die Verzahnungen innerhalb der Hirn- und Computerwissenschaften: bei der Entwicklung ihrer Theorien der natürlichen und künstlichen neuronalen Netze, dem Bau von Künstlicher Intelligenz (künftig: KI), von bildgebenden Verfahren in der Hirnforschung, von Neuroimplantaten, Gehirn-Maschine-Schnittstellen. Die Kooperationen der NBIC-Wissenschaften und -Technologien werden seit der Jahrtausendwende in den Ländern des globalen Nordens politisch stark gefördert (vgl. ebd., 79-214). Dabei standen insbesondere die US-amerikanischen NBIC-Initiativen, die Anfang der Jahrtausendwende unter maßgeblicher Beteiligung der National Science Foundation, des Handelsministeriums und Teilen der Militärforschung ergriffen wurden, unter dem Vorzeichen transhumanistischer Utopien (vgl. ebd., 109-119). Mit Hilfe dieser neuartigen Technologien sollten das individuelle Mensch-Sein optimiert, ökonomisches Wachstum generiert und nationale Sicherheit garantiert werden. Innerhalb der NBIC-Wissenschaften und -Technologien sollten Projekte in der Landwirtschaft, im Verkehr, der Produktion und dem Militär verfolgt, neue Stützpunkte im Weltraum aufgebaut, Eingriffe in die neuronalen Prozesse von Menschen und in das Genom von Menschen, Tieren, Pflanzen vorgenommen werden.

Ә OpenAccess. (C) 2021 Olivia Mitscherlich-Schoennherr, published by De Gruyter. (cc))BY-NC-ND This work is licensed under the Creative Commons Attribution 4.0 International License. 
Zum Entstehungszeitpunkt des Bandes wurden für die Öffentlichkeit die gesamtgesellschaftlichen Weichen erfahrbar, die mit der breit angelegten Förderung der konvergierenden Nano-, Bio-, Info- und Kognitionswissenschaften gestellt worden sind. Die getroffenen Weichenstellungen wurden ablesbar an Berichten über die Erfolge bei der Entwicklung neuartiger Technologien - wie technischer Pflegeassistenzsysteme und Mensch-Maschine-Schnittstellen -, über die Durchführung neuer NBIC-Projekte - wie der Mars-Mission der NASA oder der Erzeugung von Embryonen aus Affen- und Menschenzellen - sowie an den Expert_innen-Anhörungen und Stellungnahmen von Ethikräten zu ebendiesen Forschungsvorhaben (vgl. etwa Deutscher Ethikrat 2020; 2021).

Vor allem wurde die Bedeutung der NBIC-Initiativen im Entstehungszeitraum des vorliegenden Bandes jedoch an den politischen und sozialen Praktiken zur Bewältigung der gesamtgesellschaftlichen Krisen unter der Corona-Pandemie offenbar. Mit ihren Maßnahmen zur Bekämpfung der Corona-Krise hat die politische Exekutive eine evidenzbasierte Politik ausgebildet: eine politische Praxis, die ihre politischen Entscheidungen mit dem Faktenwissen über die Pandemie gerechtfertigt hat, das in empirischer Forschung erreicht wurde. Der Einfluss der NBIC-Wissenschaften auf diese evidenzbasierte Politik ließ sich an der Zusammensetzung der Gremien ablesen, von denen sich die politische Exekutive bei ihren Analysen wissenschaftlich hat beraten lassen. Vom Beginn der Corona-Krise bis in das Frühjahr 2021 wurden in diese wissenschaftlichen Beratungsgremien primär Vertreter_innen der Biowissenschaften berufen: der Virologie, Epidemiologie, Mikrobiologie, Biophysik und bestimmten Fachrichtungen der Medizin. Die Bedeutung der Konvergenzentwicklung wurde nicht nur daran sichtbar, dass die politisch zurate gezogenen Biowissenschaftler_innen ihre empirische Erforschung des gesamtgesellschaftlichen Lebens in der Pandemie mit Hilfe von KI verfolgt haben; sie hat sich auch an den inhaltlichen Analysen gezeigt. So hat etwa Claudia Priesemann, die als Physikerin in der Hirnforschung arbeitet, in der Corona-Krise als Berater_in der Bundesregierung tätig war und für ihre Forschung zur Corona-Pandemie von der Max Planck-Gesellschaft ausgezeichnet worden ist, ihre einflussreiche epidemiologische Theorie über die Ausbreitung der Pandemie entwickelt, indem sie Theorien über die Informationsausbreitung in künstlichen und natürlichen neuronalen Netzen auf die Ausbreitung des Virus in der Bevölkerung übertragen hat (vgl. Max-Planck-Gesellschaft 2021a; 2021b). Genauso wie die politischen standen auch die sozialen Praktiken, die u.a. im Gesundheitswesen zur Pandemiebekämpfung ergriffen worden sind, unter dem Einfluss der NBIC-Wissenschaften. Man denke etwa an die KI-basierte Entwicklung von Corona-Impfstoffen, Medikamenten, Diagnose-Apps, die KI-basierte Verteilung von Schutzmaterialien, die breite Etablierung von Telemedizin und die Kontaktverfolgung mit Hilfe von Tracing-Apps. 
Zugleich sind neben den Erfolgen der NBIC-Wissenschaften und -Technologien im Entstehungszeitraum des vorliegenden Bandes auch die Ambivalenzen ihrer breit angelegten Förderung und Nutzung zutage getreten. So ist etwa mit der Etablierung von Formen der Telemedizin im Zuge der Pandemiebekämpfung zugleich auch der Preis spürbar geworden, den die Digitalisierung der pflegerischen und medizinischen Begleitung abverlangt: leibliche Nähe - leibliche Berührungen, leibliches Ausdrucksverstehen - nicht oder nur in äußerst begrenztem Maße herstellen und auf diese Weise neue Formen der Entfremdung in der Begleitung generieren zu können.

Darüber hinaus ist der sozio-kulturelle Einfluss von transhumanistischen Utopien spürbar geworden, die ihre Attraktivität aus den unterschiedlichen Formen der Entgrenzung von Natur und Technik durch die NBIC-Wissenschaften und -Technologien ziehen. Transhumanistische Gedanken wurden zum Entstehungszeitpunkt des Bandes nicht nur in radikalen Versionen einer Erneuerung oder Ersetzung des Menschen durch Digitaltechnologien von schillernden Figuren wie Elon Musk vertreten, denen es immer auch um öffentliche Aufmerksamkeit und das Bedienen ihrer Investitor_innen geht. In gemäßigteren Versionen wirkten transhumanistische Vorstellungen vielmehr bis weit in die seriöse Politik und Wissenschaft hinein. Dies hat sich etwa an einem - vergleichsweise - unspektakulären Ereignis gezeigt, das ebenfalls in den Entstehungszeitraum des vorliegenden Bandes gefallen ist: an der Expert_innen-Anhörung des Deutschen Ethikrats zu KI und Mensch-Maschine-Schnittstellen (vgl. Deutscher Ethikrat 2021). Ein zentrales Anliegen dieser Anhörung hat der Möglichkeit von ,starker $\mathrm{KI}^{\prime}$ gegolten: der Frage, ob es in absehbarer Zukunft möglich werde, KI zu konstruieren, die in dem Sinne ,stark 'sei, dass sie die intelligenten Fähigkeiten von Menschen ausüben und damit in wirkliche Interaktionen mit Menschen eintreten könne. Die geladenen Expert_innen aus den Neurowissenschaften, der Theorie des maschinellen Lernens und der kognitiven Systeme sind allesamt davon ausgegangen, dass sich solche ,starke KI' künftig werde bauen lassen. Der Einfluss des utopischen Transhumanismus auf die seriöse Wissenschaft wird an dem Umstand deutlich, dass die versammelten Expert_innen der NBIC-Wissenschaften diese Annahme im Wissen über die Grenzen ihres gegenwärtigen Wissens vertreten haben: sie haben angegeben, zum Zeitpunkt ihrer Stellungnahmen noch über kein Wissen darüber zu verfügen, wie solche ,starke KI‘ künftig gebaut werden könne. Bei ihren Annahmen, dass menschengleiche künstliche Intelligenz gebaut werden könne, vertrauten sie vielmehr auf die Eigendynamik der KI-basierten KIEntwicklung, von der sie kein Wissen hatten (vgl. ebd. 9, 19, 26, 34).

Schließlich sind die Ambivalenzen der NBIC-Förderung im Entstehungszeitraum des Bandes auch an den normativen Widersprüchen der evidenzbasierten ,Corona-Politik` zutage getreten, die die politische Exekutive unter der - oben 
skizzierten - Anleitung der Biowissenschaften ausgeübt hat. ${ }^{1}$ Im Verlauf der Corona-Krise hat sich das normative Leitbild als in sich widersprüchlich herausgestellt, dem die evidenzbasierte ,Corona-Politik verpflichtet war: das Ideal einer politischen Praxis, die rationaler sein sollte als politisches Klugheitswissen bzw. ein reflektiertes Beurteilen der konkreten Handlungssituation, ${ }^{2}$ indem sie auf Basis von empirischem Tatsachenwissen die einzig richtigen politischen Entscheidungen trifft. In Widersprüche hat sich die evidenzbasierte Corona-Politik verstrickt, da sie dieses Ideal rationaler Politik realisiert hat, indem sie bei der Analyse der Corona-Krise die biowissenschaftliche Expertise überdehnt und sozial- und geisteswissenschaftliche Erkenntnisse abgeblendet hat.

In wissenschaftstheoretischer Hinsicht hat die politische Exekutive bei der Ableitung ihrer politischen Entscheidungen aus den Ergebnissen der biowissenschaftlichen Forschung nämlich die Abstraktheit der empirischen Forschungsergebnisse unterschätzt: dass die biowissenschaftlich erreichten Evidenzen nicht unmittelbar von den Sachen selbst abgelesen, sondern vielmehr in komplexen Prozesse der empirischen Forschung erreicht werden - und damit valides Wissen nur über den herausdestillierten Lebensaspekt vermitteln. Indem die evidenzbasierte Politik ihre politischen Entscheidungen mit den biowissenschaftlichen Erkenntnissen über die Corona-Pandemie begründet hat, wurde sie irrational, weil sie den Bezug der zugrunde gelegten Evidenzen auf die Erkenntnisperspektiven abgeblendet hat, in denen sie erreicht wurden - und die empirischen Evidenzen für wahres Wissen über die Corona-Krise selbst genommen hat.

Einhergehend mit dieser Überdehnung des empirischen Wissens sind der evidenzbasierten ,Corona-Politik` reduktionistische Verkürzungen bei der inhaltlichen Deutung der Corona-Krise unterlaufen. Indem sie wertneutrale empirische Evidenzen zur Grundlage ihrer politischen Entscheidungen gemacht hat, hat diese politische Praxis das nicht-quantifizierbare, wertgebundene Wissen der Geistes- und Sozialwissenschaften über das gesamtgesellschaftliche Leben in der Corona-Krise von vornherein aus ihren Analysen ausgegrenzt. Deutliches Symptom dieser Ausgrenzung der genannten Wissensbestände aus ihren Analysen der Krise war der Umstand, dass die politische Exekutive in ihre wissenschaftlichen Beratungsgremien neben den Vertreter_innen der Biowissenschaften keine Vertreter_innen der Sozial- und Geisteswissenschaften berufen hat. Inhaltlich wurde damit etwa das Wissen der Kinder- und Jugendpsychologie und der Gerontologie über psycho-soziale Vulnerabilitäten von Kindern oder Bewohnern in Pflegeein-

1 Zum Verständnis von internen Widersprüchen sozialer Praktiken und ihrer theoretischen Kritik vgl. Jaeggi 2014, 261-301; 356-391.

2 Zum Verständnis des reflektierenden Urteilens als politisches Erkenntnisvermögen vgl. Arendt 2012. 
richtungen oder das Wissen der Soziologie über die modernen - auf Wachstum gestellten - Gesellschaften abgeblendet, in deren Rahmen sich die Pandemie ereignet hat und deren Eigendynamiken eigene Gefahren hervorgebracht haben: etwa die besonderen Gefährdungen durch das Virus für Menschen, die in prekären Beschäftigungsverhältnissen arbeiten oder außerhalb des globalen Nordens leben.

Nur vor dem Hintergrund dieser wissenschaftstheoretischen und inhaltlichen Verkürzungen konnte die politische Exekutive bei ihren Analysen davon ausgehen, dass sich die Corona-Krise im Ausgang von den - quantitativ messbaren biologischen Prozessen der Pandemie abschließend bestimmen lasse. Die (scheinbaren) Alternativlosigkeiten im politischen Entscheiden haben sich damit nicht - wie von der evidenzbasierten Politik beansprucht - aus der wahren Analyse der Krise gespeist, sondern vielmehr aus der fehlenden Reflexion auf die Praktiken, in denen die biowissenschaftliche Perspektive zur einzig richtigen erhoben wurde. Damit hat sich zugleich gezeigt, dass die evidenzbasierte CoronaPolitik im Widerspruch zu ihrem eigenen Anspruch auf werturteilsfreies politisches Handeln ihrerseits Wertbindungen etabliert hat, ohne dies zu reflektieren: die Bindungen der politischen Krisenbewältigung an die NBIC-Wissenschaften als gesamtgesellschaftliche ,Leitwissenschaften“ und mittelbar damit zugleich an den Wert des Lebensschutzes als maßgeblicher Norm.

\section{Zu Gegenstand, Fragestellung und methodischer Anlage des Bandes}

Indem der vorliegende Band nach dem Mensch-Sein an den Grenzen des Lebens unter Bedingungen disruptiver Biotechnologien fragt, wählt er einen besonderen Fokus auf die NBIC-Wissenschaften und -Technologien. Er fokussiert auf solche Technologien, mit deren Hilfe in das körper-leibliche bzw. personale Leben von Menschen eingegriffen wird und dessen Grenzen in irgendeinem Sinne verschoben werden: auf neuartige Pränatal-, Neuro- und Gerotechnologien. Andere NBICTechnologien und -Projekte, die gesellschaftlich nicht minder relevant sind und ebenfalls bereits Gegenstand der US-amerikanischen Forschungsinitiativen von 2001 waren, grenzt der Band dagegen aus: etwa KI-basierte Projekte in der Landwirtschaft oder neue Weltraum- und Militärprojekte.

In seiner Auseinandersetzung mit neuartigen Lebensformen mit disruptiven Biotechnologien bewegt sich der Band auf unterschiedlichen Ebenen. In einer ersten Hinsicht setzt er sich mit neuen Formen der Existenz an den Grenzen des Lebens - am Lebensanfang, am Lebensende und in Krisensituationen - im 
Rahmen von Therapie- und Pflegebeziehungen mit neuartigen Biotechnologien auseinander. In einer zweiten Hinsicht fragt er nach Praktiken der Züchtung und des Enhancements bzw. der Leistungssteigerung von gesunden Menschen mit Hilfe von Biotechnologien. Und in einer dritten Hinsicht blickt er schließlich auf die trans- und posthumanistischen Utopien einer biotechnologischen Optimierung des Geistes, die die Grenzen der menschlichen Lebensform transzendiert. Der Band verbindet diese unterschiedlichen Ebenen der Auseinandersetzung in der Überzeugung, den Transformationen des Mensch-Seins nur mit Blick auf diese unterschiedlichen Technikverhältnisse gerecht werden zu können, die sich bei der Entwicklung und Nutzung disruptiver Biotechnologien durchdringen.

Wenn der Band die thematisierten Biotechnologien als disruptiv kennzeichnet, dann greift er auf einen Begriff zurück, der ursprünglich von dem Wirtschaftswissenschaftler Clayton M. Christensen geformt worden ist. Im Deutschen wurde dessen Ausdruck der „disruptive innovations“ zunächst als „,bahnbrechende Innovationen“ übersetzt (vgl. Christensen 2011). Wie Christensen nimmt der Band eine pragmatistische Perspektive auf die Technologien ein und thematisiert sie in ihrer Verankerung in sozio-kulturellen Praktiken. Der disruptive Charakter entscheidet sich dergestalt nicht für sich genommen an den technologischen Innovationen, die die wissenschaftlich-technischen Konvergenzen ermöglichen, sondern an den sozio-kulturellen Praktiken, die mit den neuartigen Konvergenztechnologien möglich werden. Über Christensens ökonomisch verengte Perspektive auf den disruptiven Status technologischer Innovationen geht der Band jedoch hinaus. Im Unterschied zu Christensen versteht er technologische Innovationen nicht dann als disruptiv, wenn sie ökonomisches Wachstum generieren, sondern wenn sie bisher nicht gekannte Formen menschlichen Lebens eröffnen. Dabei werden auf den drei Ebenen der Therapie, des Enhancements und des Trans- bzw. Posthumanismus nicht nur unterschiedliche Technologien behandelt; es kommen auch unterschiedliche Menschenbilder und Vorstellungen über die Grenzen menschlichen Lebens und das Ineinandergreifen von Technologie und menschlicher Natur in den Blick; und es werden unterschiedliche Formen des biotechnologisch vermittelten Durchbrechens der Grenzen des Lebens diskutiert.

Methodisch führt der Band die Auseinandersetzung mit dem Leben mit disruptiven Biotechnologien in transdisziplinärer Perspektive. Er versammelt Beiträge aus unterschiedlichen Strömungen der philosophischen Anthropologie, Ethik und politischen Philosophie, aus unterschiedlichen Subdisziplinen der Medizin, aus der Medizinethik, den Pflegewissenschaften und der Pflegeethik sowie aus den Politikwissenschaften. Das Scharnier im transdisziplinären Dialog benennt im Titel des Bandes das Konzept der ,künstlichen Natürlichkeit‘. Dieses Konzept bringt die anti-dualistische Perspektive auf das Mensch-Sein mit dis- 
ruptiven Technologien zum Ausdruck, die die versammelten Beiträge in all ihrer methodischen und inhaltlichen Vielfältigkeit teilen.

Unter dem Konzept der ,künstlichen Natürlichkeit‘ sind die Aufsätze über disruptive Technologien der Therapie, des Enhancements und der trans- bzw. posthumanistischen Überwindung des Mensch-Seins auf der einen Seite in der Distanz zu naturalistischen Anthropologien vereint, die unter Vertreter_innen der NBIC-Technologien verbreitet sind. Solche naturalistischen Anthropologien beschränken die menschliche Natur auf Organ- und insbesondere Hirnfunktionen. Das psycho-soziale Leben von Menschen wird auf ein bloßes Epiphänomen der biologischen Prozesse reduziert. So sollen in naturalistischer Perspektive etwa Gefühlszustände, Stimmungen, Emotionen von den Hirnaktivitäten abgelesen werden. Vor dem Hintergrund solcher naturalistischen Verkürzungen wird der Anspruch erhoben, mit biotechnologischen Eingriffen in biologische Prozesse in das menschliche Erbgut, molekularbiologische Prozesse, Hirnfunktionen eine Veränderung, Optimierung oder Transzendierung der menschlichen Natur direkt bewirken zu können. Der vorliegende Band lässt solche naturalistischen Anthropologien im Wissen hinter sich, dass die Reduktion des psycho-sozialen Lebens auf Epiphänomene biologischer Prozesse weder dem erst- und zweitpersonalen Erleben noch dem sozio-kulturellen Miteinander und dem Status von Technik gerecht wird.

Auf der anderen Seite markiert das Konzept der ,künstlichen Natürlichkeit“ die Distanz des Bandes zu rationalistischen oder konstruktivistischen Menschenbildern. Solche rationalistisch-konstruktivistischen Anthropologien beschränken die menschliche Natur bzw. die menschliche Lebensform auf soziokulturelle Praktiken und Bilder. Komplementär zu den naturalistischen Reduktionismen werden das körper-leibliche Leben und Erleben von Menschen nun auf Epiphänomene sozio-kultureller Prozesse verkürzt. So soll aus dieser konstruktivistischen Perspektive etwa der Tod eines Menschen an den Verhaltensweisen wie Explantieren, Beerdigen, Betrauern - abgelesen werden, die seine Mitwelt ihm entgegenträgt. Der vorliegende Band nimmt Distanz von solchen rationalistisch-konstruktivistischen Anthropologien, da die Reduktion des körper-leiblichen Lebens und Erlebens auf Epiphänomene sozio-kultureller Prozesse sowohl an der Eigendynamik biologischer Prozesse als auch am erst- und zweitpersonalen Erleben - Erfahrungen des eigenen Leibes, des Anderen - vorbeigeht.

Unter dem Konzept der ,künstlichen Natürlichkeit‘ schlägt der Band aus den Einsichten in die Grenzen der naturalistischen und rationalistisch-kulturalistischen Reduktionismen nun allerdings auch kein Kapital für eine Verabsolutierung des unmittelbar leiblichen Erlebens: für solche Spielarten der Leibesphänomenologie, die das unmittelbare leibliche Leben und Erleben zum eigentlichen Mensch-Sein überhöhen, um biotechnologische Eingriffe als Formen der Ent- 
fremdung von der menschlichen Natur zu kritisieren. Abgeblendet wird in solchen Entfremdungstheorien nämlich nicht nur die motivationale Einsicht, dass in der Gegenwart wohl kaum jemand ganz auf die neuen Lebensmöglichkeiten verzichten mag, die die biotechnologische Medizin und Pflege eröffnen; übersehen wird darin vielmehr zugleich auch die anthropologische Erkenntnis, dass das unmittelbare leibliche Leben und Erleben in körperliche Organprozesse verschränkt und durch Kultur, Zivilisation und Technik vermittelt ist. In Distanz zu solchen Mythen der Unmittelbarkeit setzt sich der vorliegende Band vielmehr mit realisierten, angestrebten oder erträumten Formen menschlichen Lebens mit disruptiven Biotechnologien auseinander, in denen biologische Prozesse, leibliches und emotionales Erleben und sozio-kulturelle Praktiken und Bilder auf vielfältige Weise ineinandergreifen. In den Beiträgen zum Band werden die anthropologischen, ethischen und politischen Implikationen von neuartigen Lebensformen diskutiert, die mit disruptiven Biotechnologien ausgeübt werden (sollen), und in denen die Grenzen des menschlichen Lebens durchbrochen werden (sollen), das wir bisher kannten.

Mit der Frage nach dem Gelingen der künstlichen Natürlichkeit ist im Titel des Bandes schließlich sein normativer Einsatz markiert. Die Gelingensfrage verweist auf das Anliegen der Aufklärung, dem der Band verpflichtet ist. Jenseits von Technikvergottung und Technikverteufelung will er aus transdisziplinärer Perspektive zu Mündigkeit bei der Entwicklung, Zulassung und Nutzung disruptiver Biotechnologien beitragen. Dabei wählen die Autor_innen zum einen unterschiedliche Formen der Kritik. Im Band wird nicht nur Kritik an den naturalistischen Reduktionismen der transhumanistischen Utopien der Gegenwart geübt. Es werden auch die normalisierenden Festlegungen kritisiert, die die Nutzer_innen von bestimmten Pränatal-, Neuro- und Gerotechnologien erfahren können und die sich ihrerseits aus den Menschenbildern und ethischen Vorannahmen über ein gelingendes Leben speisen, die deren Macher_innen in diese Biotechnologien eingebaut haben. So werden etwa die Festlegungen einer Kritik unterzogen, die das Leben im Alter und die Altenpflege durch die Altersbilder erfahren können, die in technische Pflegeassistenzsysteme eingebaut werden. In Ergänzung zu solchen kritisch angelegten Aufsätzen gehen einige Autor_innen zum anderen in affirmativer Hinsicht den Fragen nach solchen Formen des technologisch vermittelten Mensch-Seins nach, in denen die Nutzer_innen durch die Modellierung der Biotechnologien nicht normalisierend festgelegt, sondern vielmehr zu pluralen Formen selbstbestimmten Lebens befähigt werden. 


\section{Zum inhaltlichen Aufbau des Bandes}

In seinen drei Teilen thematisiert der Band die Transformationen des menschlichen Lebens durch die Nutzung disruptiver Biotechnologien in den oben unterschiedenen Hinsichten: die neu hervorgebrachten Existenzformen an den Grenzen des Lebens in Therapie- und Pflegebeziehungen mit disruptiven Pränatal-, Neuro- und Gerotechnologien, die neuartigen Formen der biotechnologischen Züchtung und Optimierung menschlichen Lebens und die Utopien eines transund posthumanen Lebens, das in technologisch kontrollierter Evolution hervorgebracht werden soll. Aufgrund seiner Breite muss der Band notwendigerweise Konzessionen bei der Ausführlichkeit der Auseinandersetzung mit seinen einzelnen Unterthemen machen und kann jeweils nur aussagekräftige Schlaglichter setzen.

\subsection{Menschliche Existenz in Therapie- und Pflegebeziehungen mit disruptiven Biotechnologien}

In seinem ersten Teil rückt der Band die menschliche Existenz an den Grenzen des Lebens in den Fokus: am Lebensanfang, am Lebensende und in Krisensituationen schwerer Erkrankung. Da die menschliche Existenz an den Grenzen des Lebens in den Ländern des globalen Nordens in das Gesundheitswesen eingebettet ist, nimmt er zusammen mit dem Gebären, Altern und Sterben sowie dem Leben mit schwerer Erkrankung auch deren Begleitung in Pflege- und Therapiebeziehungen in den Blick. Als neuartige Formen menschlicher Existenz setzen sich die Beiträge zu diesem ersten Schwerpunkt dementsprechend mit bisher nicht gekannten Formen des Gebärens, Alterns und Sterbens sowie des Lebens mit schwerer Erkrankung im Kontext von Therapie- und Pflegebeziehungen mit disruptiven Pränatal-, Gero- und Neurotechnologien auseinander. Dabei werden diese neuartigen Formen der Begleitung und der Existenz von den disruptiven Biotechnologien ermöglicht und in grundlegender Weise durchdrungen.

Der Band macht seinen Anfang mit dem Anfang des menschlichen Lebens: mit dem Gebären und der Geburtshilfe mit disruptiven Pränataltechnologien. Dabei wirft er ein exemplarisches Schlaglicht auf eine besondere Gestalt der Kinderwunschbehandlung und der reproduktionsmedizinisch ermöglichten Mutterschaft: auf die Leihmutterschaft, die in Deutschland verboten, in anderen Ländern innerhalb und außerhalb der EU dagegen erlaubt ist. ${ }^{3}$ Für die Auseinandersetzung mit dem bio-

3 In Ergänzung sei auf den Schwerpunkt über Bioethik und Biopolitik der Pränataldiagnostik im 
technologisch vermitteltem Lebensanfang ist die Leihmutterschaft von besonderem Interesse. Als therapeutische Praxis zur Behandlung von Sterilität nimmt die Leihmutterschaft disruptive Reproduktions- und Pränataltechnologien wie künstliche Befruchtung und vorgeburtliche Diagnostik in Anspruch. Zugleich wird das menschliche Gebären im Rahmen dieser therapeutischen Praxis grundlegend revidiert. Es wird nicht nur technologisch in die körper-leiblichen Prozesse der Befruchtung und Einnistung eingegriffen; auch das Eltern-Kind-Verhältnis wird grundlegend umgestaltet zu der ,Dreiecksbeziehung“ von Kind, austragender Frau und beauftragendem Paar. An den Eltern-Kind-Verhältnissen, die mit der Kinderwunschbehandlung der Leihmutterschaft etabliert werden, entzünden sich ethische und politische Debatten.

Im ersten Aufsatz des Bandes diskutiert Heribert Kentenich aus reproduktionsmedizinischer, psychotherapeutischer und medizinethischer Perspektive das Statement aus dem Koalitionsvertrag der großen Koalition aus dem Jahr 2013 zur Leihmutterschaft: dass Leihmutterschaft als mit der Würde des Menschen unvereinbar abzulehnen sei. Um diese These zu überprüfen, ordnet der Autor die Leihmutterschaft zunächst in den Kontext der Kinderwunschbehandlung ein und führt aus psychotherapeutischer Sicht eine differenzierte Auseinandersetzung mit dem Leiden an nicht erfülltem Kinderwunsch. In seiner Auseinandersetzung mit der Leihmutterschaft als Sterilitätstherapie für Frauen ohne Gebärmutter oder ohne funktionsfähige Gebärmutterschleimhaut kommt Kentenich - ähnlich wie die Leopoldina in ihrer Stellungnahme zu einer zeitgenössischen Fortpflanzungsmedizin - zu einer ambivalenten Einschätzung (vgl. Leopoldina 2019, 7885). Vor diesem Hintergrund sind die behandelnden Ärzt_innen nach Kentenich mit hohen Anforderungen an eine umfassende juristische, psychotherapeutische und ethische Beratung konfrontiert.

In Ergänzung zu Kentenich setzt sich Anca Gheaus in ihrem Beitrag mit Leihmutterschaftsverträgen aus der Perspektive der philosophischen Ethik auseinander. Gheaus fragt angesichts von Fällen, in denen Leihmütter nach der Entbindung das Kind an das auftraggebende Paar nicht abgeben wollen, nach der moralischen Legitimität von Leihmutterschaftsverträgen. In ihren ethischen Überlegungen geht sie von phänomenologischen Studien über die Bindungen aus, die während der Schwangerschaft zwischen der Schwangeren und dem Kind entstehen. Vor dem Hintergrund dieser pränatalen Bindungen tritt sie dafür ein, dass der gebärenden Mutter das moralische Recht zukomme, das von ihr geborene Kind aufzuziehen. Rechtsphilosophisch fordert sie aus diesem Grund, Leihmut-

Band „Gelingende Geburt“ verwiesen, den ich zusammen mit Reiner Anselm herausgegeben habe; vgl. Mitscherlich-Schönherr/Anselm 2021, 273-339. 
terschaftsverträge bei Konflikten über die Abgabe des Kindes nach der Entbindung als nichtig anzusehen.

Der Band setzt seine Auseinandersetzungen mit der menschlichen Existenz unter Bedingungen disruptiver Biotechnologien mit Blick auf das Lebensende fort. Am Lebensende konzentriert er sich auf das Leben im Alter in Pflegebeziehungen mit disruptiven Gerotechnologien. An der Entwicklung und dem Einsatz von Gerotechnologien haben sich insbesondere angesichts der besonderen Vulnerabilität alter Menschen, den Gefahren der Vereinsamung im Alter und der Bedeutung von Interleiblichkeit für gute Pflegebeziehungen ethische Debatten entzündet. Im vorliegenden Band zeigt Mark Schweda aus der Perspektive philosophischer Anthropologie, dass in den gegenwärtigen Auseinandersetzungen über die Modellierung von Gerotechnologien Bilder und Praktiken menschlichen Lebens im Alter mitverhandelt werden. In seinem Aufsatz gibt Schweda zunächst einen Gesamtüberblick über den aktuellen Stand der Gerotechnologien, von neuartigen Modellen des Hausnotrufknopfs über Gesundheits-Apps bis zu technischen Pflegeassistenzsystemen - den sogenannten ,Pflegerobotern“ - und umfassenden Smart Home-Anlagen. Vor diesem Hintergrund leistet er nicht nur eine kritische Reflexion der Vorstellungen über menschliches Altern und seinen Status für das menschliche Leben, die in diesen Gerotechnologien in Anspruch genommen werden. Er zeigt darüber hinaus auch, dass unter der Anwendung dieser Technologien die eingebauten Annahmen über das Altern das Leben von älteren Menschen und die gesellschaftlichen Vorstellungen über ältere Menschen mitbestimmen.

In Ergänzung zu Schweda konzentriert sich Constanze Giese auf KI-basierte Pflegeassistenzsysteme. Aus den Perspektiven der Pflegewissenschaft und der Pflegeethik fragt Giese nach den Anforderungen an gute Pflege mit ebendiesen neuartigen technischen Systemen. Dafür entwickelt sie zum einen in deskriptiver Hinsicht ein differenziertes Verständnis von den pflegerischen Handlungsfeldern, in denen technische Assistenzsysteme zur Anwendung kommen können, sowie vom Setting der Nutzung und den potenziellen Nutzer_innen: von unterstützungsbedürftigen Personen über Angehörige bis zum Pflegefach- und Pflegehilfspersonal. In normativer Hinsicht skizziert sie zum anderen die Standards guter Pflege, die in den zeitgenössischen Pflegewissenschaften entwickelt worden sind. Vor dem Hintergrund dieser deskriptiven und normativen Wissensbestände wird es ihr am Ende ihrer Überlegungen möglich, Anforderungen an die Bildung der Pflegenden und an die Forschung, Entwicklung sowie den Einsatz von technischen Pflegeassistenzsystemen zu formulieren, um mit ihrer Hilfe gute Pflege zu gewährleisten.

Zum Abschluss seines ersten Teils wendet sich der Band Krisensituationen der personalen Existenz und deren therapeutischen Behandlung zu. Dabei kon- 
zentriert er sich auf Krisen schwerer neurologischer bzw. psychologischer Erkrankung und deren neurologische bzw. psychiatrische Therapien. Aufgrund dieser Schwerpunktsetzung rücken in technischer Hinsicht insbesondere disruptive Neurotechnologien und unter den Neurotechnologien wiederum die Tiefe Hirnstimulation in den Fokus des Interesses, mit denen neuronale bzw. zerebrale Funktionen manipuliert werden. Unter der Behandlung mit Neuroprothesen kommt es zu bisher nicht gekannten Formen des Ineinandergreifens der Technologien und der personalen Existenz ihrer Nutzer_innen: der behandelnden Ärzt_innen und der behandelten Patient_innen. Hieran entzünden sich Fragen nach den Menschenbildern und den Vorstellungen guter Medizin und gelingenden Lebens, die in die Neuroprothesen eingebaut werden, sowie nach der Konstruktion von guten Neuroprothesen und einer verantwortlichen Technikentwicklung.

Tobias Sitter geht in seinen Überlegungen von dem Phänomen aus, dass neurotechnologische Eingriffe in Hirnfunktionen häufig mit Veränderungen des psycho-sozialen Lebens der Betroffenen einhergehen. Angesichts solcher Korrelationen erarbeitet Sitter einen Reflexionsrahmen philosophischer Anthropologie, um die neurotechnologische Einflussnahme auf menschliche Lebensvollzüge mitsamt ihren psycho-sozialen Aspekten verstehen und konkrete Anwendungsfälle differenziert beurteilen zu können. In kritischer Absicht zeigt er, dass sich verbreitete Ansätze eines reduktiven Naturalismus zur Bewältigung dieser Aufgabe nicht eignen. In affirmativer Absicht entwickelt er eine Theorie konkreter Subjektivität, die die Form menschlichen Lebens in einer binnendifferenzierten Einheit findet, in der Bewusstsein und Organismus als zwei nicht aufeinander reduzierbare Aspekte verschränkt sind. Anhand ausgewählter Fallbeispiele zeigt Sitter, dass sich diese anthropologische Theorie als Reflexionsrahmen eignet, um neurotechnologische Einflussnahmen auf das menschliche Leben in all seinen körper-leiblichen wie psycho-sozialen - Aspekten differenziert zu beurteilen.

In meinem eigenen Aufsatz beschäftige ich mich aus der Perspektive eines personalen Ansatzes innerhalb der philosophischen Anthropologie mit der Entwicklung und Anwendung einer bestimmten Neuroprothese: mit Tiefer Hirnstimulation (THS) mit geschlossenem Regelkreis bzw. ,closed loop‘. Obgleich die technologische Entwicklung dieser algorithmen-basierten Systeme erst am Anfang steht, ist vor dem Hintergrund der Technikentwicklung der vergangenen Jahre die Konstruktion von solchen THS-Systemen mit ,closed loop'-Verfahren absehbar, in denen das Implantat als autopoetisches System funktioniert und die Anpassung der therapeutischen Stimulierung auf Basis der gemessenen Hirnfunktionen vornimmt. Um einen theoretischen Beitrag zur Entwicklung von solchen Systemen zu leisten, in denen die technischen Möglichkeiten des ,closed loop'-Verfahren genutzt werden, ohne dafür den Preis normalisierender Festle- 
gungen ihrer Nutzer_innen zu zahlen, setze ich mich kritisch mit den grundlegenden Optionen auseinander, gute THS mit ,closed loop` zu bauen. Die Systeme können so gebaut werden, dass eine gute Diagnostik und Therapieanpassung während der Behandlung entweder durch den Algorithmus unter Umgehung der personalen Therapiebeziehung oder durch Einbau von Rückkoppelungsschleifen an die personale Therapiebeziehung sichergestellt werden sollen. Beide Optionen guter THS befrage ich in Bezug auf ihre ethischen und anthropologischen Voraussetzungen und ihre Implikationen für das Leben der Nutzer_innen - und votiere für den Bau von hybriden Modellen, in denen mit Hilfe einer eingebauten Alarmfunktion die Letztverantwortung für Diagnostik und Therapie unter der THS-Behandlung der personalen Therapiebeziehung überlassen bleibt.

Aus einer technikethischen und -politischen Perspektive fragt Christoph Kehl im dritten Aufsatz über disruptive Neurotechnologien schließlich nach den Möglichkeiten einer verantwortungsvollen Gestaltung und weiteren Entwicklung dieser Technologien. Dafür leistet er zunächst einen kritischen Abgleich der utopischen Visionen von Mensch-Maschine-Entgrenzungen durch disruptive Neurotechnologien mit den tatsächlichen Realitäten der Technologieentwicklung. In Anschluss daran skizziert er die Herausforderungen, die sich an eine vorausschauende Technikgestaltung aus den Spannungen von utopischen Spekulationen und einem relativ frühen Stand der Technologieentwicklung ergeben. Vor dem Hintergrund dieser Herausforderungen identifiziert Kehl grundsätzliche Ansatzpunkte einer ethischen Technikgestaltung und diskutiert deren Möglichkeiten und Grenzen. Dabei arbeitet er auch die zwiespältige Rolle von professioneller Ethik im Rahmen neuerer Ansätze zur Technology Governance zwischen Ethics Washing und Gestaltungsanspruch heraus.

\subsection{Biotechnologische Optimierung des menschlichen Lebens: Züchtung und Enhancement}

Während die Beiträge zum ersten Teil sich mit der Nutzung von Biotechnologien zu Zwecken der Therapie und Pflege auseinandergesetzt haben, beschäftigen sich die Beiträge zum zweiten Teil mit ihrer Nutzung zu Zwecken der Züchtung bzw. des Enhancements von gesunden Menschen. In technischer Hinsicht rücken Genund Neurotechnologien von Genome Editing über Gesundheits-Apps, Psychopharmaka, Neuroprothesen bis zu fiktiven ,Moralpillen' in den Blick. Die versammelten Aufsätze setzen sich mit den Bestrebungen auseinander, durch biotechnologische Eingriffe in biologische Prozesse das Leben von gesunden Menschen vor oder nach der Geburt zu optimieren: die Grenzen ihrer Moral-, Glücks-, Erkenntnis- und Leistungsfähigkeiten biotechnologisch herauszuschie- 
ben, die bisher als naturgegeben bzw. als durch die menschliche Lebensform mitgegeben angesehen worden sind. ${ }^{4}$ Dabei wenden sich Andreas Heinz und Assina Seitz und Björn Sydow primär den Konsequenzen von biotechnologischem Enhancement für die Einzelnen, Christina Schües und Petra Schaper-Rinkel primär seinen Konsequenzen für das intersubjektive Miteinander zu.

Björn Sydow macht sich die Verteidigung der lebensweltlichen Intuition zur Aufgabe, dass an biotechnologischem Enhancement moralisch relevanter Gefühle mit Hilfe einer fiktiven ,Moralpille، in moralischer Hinsicht etwas nicht stimme. Sydow legt seinen Aufsatz als ethischen Lernprozess an. Im Verlauf seines Aufsatzes erarbeitet er die ethischen Erkenntnisse, mit deren Hilfe er eine biotechnologische Manipulation moralisch relevanter Gefühle als moralischen Fehler aufweisen kann. In einem ersten Schritt kritisiert er Positionen als zu voraussetzungsreich, die die Möglichkeiten prinzipiell leugnen, moralisches Handeln mit biotechnologischen Eingriffen zu optimieren. In einem zweiten Schritt wendet er sich Positionen zu, die sich allein einer Verpflichtung zum biotechnologischen Enhancement der Moral widersetzen. Er zeigt, dass Positionen, die die Einnahme einer Moralpille gegenüber mühevoller Auseinandersetzung mit den eigenen Gefühlen als wirkungsvoller anerkennen, scheitern, wenn sie eine Pflicht zum biotechnologischen Enhancement der Moral mit Bezug auf die individuelle Selbstbestimmung abwehren wollen. Dafür sei ihr verkürztes Verständnis von Selbstbestimmung verantwortlich. Demgegenüber arbeitet Sydow die Bedeutung von moralisch relevanten Gefühlen für individuelle Selbstbestimmung heraus. Vor diesem Hintergrund kann er verstehen, dass die Unterdrückung von Gefühlen, die moralisches Handeln erschweren, ein moralischer Fehler ist: darin werden nämlich die Möglichkeiten von individueller Selbstbestimmung preisgeben.

Andreas Heinz und Assina Seitz setzen sich aus Perspektive der Psychiatrie mit den Gefahren von Neuroenhancement für ihre Nutzer_innen auseinander. Dabei konzentrieren sie sich auf Neuroenhancement mit Hilfe von Medikamenten. Heinz und Seitz plädieren dafür, den Einsatz von Pharmaka zur kognitiven Leistungssteigerung weder bei Kindern noch bei gesunden Erwachsenen gesetzlich zuzulassen. Zur Begründung ihrer Position verweisen sie nicht nur auf das Suchtrisiko, das mit allen derzeit verfügbaren Medikamenten zur kognitiven Leistungssteigerung verbunden ist und sich wahrscheinlich auch bei der künfti-

4 Wer sich neben diesen Bestrebungen, die menschliche Existenz mit Hilfe von Biotechnologien qualitativ zu verbessern, auch für die Bestrebungen interessiert, sie quantitativ - im Sinne eines Enhancements der Lebensspanne durch die zeitgenössischen Alternswissenschaften und -technologien - zu verbessern, sei auf den Band „Länger leben“ verwiesen, den Sebastian Knell und Marcel Weber 2009 besorgt haben. 
gen Medikamentenentwicklung nicht beheben lassen wird. Vielmehr machen sie darüber hinaus auch auf die sozio-kulturellen Kontexte einer möglichen Zulassung von Psychopharmaka zur Steigerung der Leistungsfähigkeit aufmerksam. In neoliberalen Gesellschaften könne es zum sozialen Druck auf die Einzelnen kommen, Neuroenhancement trotz seines Suchtrisikos nach einer Freigabe in Bewerbungs- und Prüfungssituationen oder im Arbeitsleben zu nutzen. In Ergänzung weisen sie Spekulationen über die positiven Effekte des Neuroenhancement auf das gesellschaftliche Gefüge zurück. Die relative Zunahme der kognitiven Leistungsfähigkeit durch Neuroenhancement würde nach Heinz und Seitz kaum zu einer Verbesserung der gesellschaftlichen Ordnung, sondern vielmehr zu einer Steigerung sozialer Ungleichheiten führen - die wiederum negative Konsequenzen für die Entwicklung kognitiver Fähigkeiten hätten.

Christina Schües und Petra Schaper-Rinkel fragen in Anschluss an Überlegungen von Hannah Arendt nach den Konsequenzen von biotechnologischen Formen der Züchtung bzw. der Verhaltenslenkung für die Grundlagen von Moral und Politik. Christina Schües blickt aus einer Perspektive der philosophischen Anthropologie auf die Konsequenzen von gentechnologisch vermittelten Formen der Züchtung für die menschliche Fähigkeit, Versprechen zu geben und zu halten. Sie zeigt, dass die menschliche Fähigkeit zum Versprechen, die sie als Grundlage von Moral versteht, damit zu tun hat, wie die Gebürtlichkeit eines Menschen als mitmenschliches Beziehungsgeschehen anerkannt wird. Angesichts der Bedeutung des relationalen Angefangen-Werdens für Mit- und Zwischenmenschlichkeit untersucht Schües unterschiedliche historische Paradigmen der Züchtung und Lenkung von Fortpflanzung. Das moderne Züchtungsparadigma zehre von der modernen Genetik, die Erbmerkmale und genetische Dispositionen kennt. Züchtung werde in der Moderne in Hinwendung an das ,biologische Substrat' von Menschen betrieben, das geteilt, eingefroren, weitergegeben, geprüft und verändert werde. Schües macht deutlich, dass das anthropotechnische Züchtungsprojekt der Moderne aufgrund seiner biologistischen Anlage Konsequenzen für die moralische Kategorie des Versprechens hat.

Petra Schaper-Rinkel extrapoliert aus Perspektive der Politikwissenschaften die Konsequenzen von Verhaltenssteuerung durch Psychopharmaka und Digitaltechnologien für die Demokratie. Die Grundlagen von Demokratie findet sie in der genuin politischen Tätigkeit des intersubjektiv geteilten Handelns. Im Miteinander-Handeln werde zwischen den Handelnden eine geteilte Welt gestiftet, die zur Ausübung von politischer Freiheit befähige. Vom Handeln unterscheidet Schaper-Rinkel das Verhalten als ein Tätig-Sein, dem politische Freiheit abgeht, da ihm der Bezug auf die mit Anderen geteilte Welt fehlt. In der Gegenwart wendet sie sich Praktiken der Verhaltenslenkung zu, die in Formen der Überwachung und Optimierung des Verhaltens mit Hilfe von Apps, Psychopharmaka, Neurofeed- 
backs, Implantaten ausgeübt werden. Unter der Nutzung solcher biotechnologischen Enhancement-Anwendungen wird nach Schaper-Rinkel der politisch zentrale Unterschied von Handeln und Verhalten diffus. Die Nutzung werde nämlich als selbstbestimmtes Handeln erfahren, in ihr setzten sich jedoch Imperative der Steuerung durch, die diesen Optimierungstechnologien eingeschrieben seien und die Nutzung zu einem gesteuerten Verhalten formten. Schaper-Rinkel kann zeigen, dass mit dem Unterschied von Handeln und Verhalten mittelbar die Welt des geteilten Handelns und damit die Grundlagen von Demokratie unterminiert werden.

\subsection{Die trans- und posthumanistischen Utopien von einer Verbesserung der menschlichen Lebensform durch technologisch kontrollierte Steuerung der Evolution}

Die Beiträge zum dritten Teil des Bandes machen sich trans- und posthumanistische Utopien zum Gegenstand, in dessen Lichte sich die Entwicklung und Anwendung der NBIC-Wissenschaften und -Technologien vollzieht. Trans- und posthumanistische Utopien werden in unterschiedlichen Spielarten und in unterschiedlicher Radikalität der technologischen Optimierung oder Überwindung des gegenwärtigen Mensch-Seins vertreten. In ihrer Verschiedenheit teilen sie das Bestreben, mit Hilfe von Algorithmen-basierten Technologien nicht nur in das Leben von gesunden Menschen, sondern vielmehr in die Evolution einzugreifen. Es geht ihnen nicht nur darum, durch Manipulation biologischer Prozesse die Leistungsfähigkeit Einzelner zu optimieren, sondern vielmehr darum, durch technologisch kontrollierte Steuerung der Evolution die menschliche Lebensform zu verbessern. Als Telos dieser Entwicklung gilt den meisten Transhumanist_innen ein unsterbliches Bewusstsein.

Tobias Müller und Hans-Peter Krüger setzen sich in den ersten beiden Beiträgen zum Schwerpunkt mit zwei Leitgedanken des Trans- und Posthumanismus auseinander: mit den fiktiven Praktiken des Mind-Uploading und dem Ideal einer Allgemeinen Künstlichen Intelligenz. Mit Hilfe des Mind-Uploading soll der Schritt in die transhumanistische Lebensform gemacht werden; in der Allgemeinen Künstlichen Intelligenz soll das transhumanistische Leben erreicht sein. Tobias Müller zeigt in seinem Aufsatz die prinzipiellen Grenzen des transhumanistischen Projekts auf, indem er die Praktiken des Mind-Uploading einer kritischen Überprüfung unterzieht. Müller kritisiert diese Praktiken im Ausgang von dem Verständnis des Bewusstseins, dem sie verpflichtet sind. Bewusstsein werde darin mechanistisch als eine Art Datenstruktur vorgestellt, die auf einen Roboter 
transferiert werden soll. Auf diese Weise soll dem Bewusstsein ein nahezu unendliches Fortbestehen im Cyperspace eröffnet werden. Müller kann jedoch zeigen, dass darin die Abstraktheit der kausal-funktionalen Beschreibung des Bewusstseins verkannt wird. Diese Beschreibung habe zwar als Beschreibung eines Teilaspekts menschlicher Subjektivität eine gewisse Berechtigung. Die konkrete Subjektivität von Lebewesen könne jedoch nicht ohne ihre Einbettung in organische Lebenszusammenhänge gedacht werden. Müller macht auf diese Weise deutlich, dass die hypothetischen Praktiken, Bewusstsein auf ein technisches System zu übertragen, bei ihrer Durchführung notwendigerweise scheitern müssen, da sie einem abstrakten Verständnis von Bewusstsein aufsitzen.

Hans-Peter Krüger wendet sich in seinem Aufsatz künstlichen neuronalen Netzwerken zu, die in Nachahmung von neuronalen Netzen im Gehirn gebaut werden. Wie jede Form der KI versteht Krüger auch diese gegenwärtig fortgeschrittenste Form von KI als maschinelles situativ-rechnerisches Problemlösen. Krüger unterscheidet nun kritisch zwischen zwei Formen des Umgangs mit künstlichen neuronalen Netzwerken. Wenn künstliche neuronale Netzwerke in die personale Lebensform von Menschen integriert werden, dann können sie von großem Nutzen sein. Den Algorithmen werde dabei das Errechnen von Mustern überantwortet, worin sie schneller und sicherer als Menschen seien. Die qualitative Deutung der errechneten Muster werde dagegen von sachverständigen Personen ausgeübt. Da sie Verstehen impliziert und sich insofern nicht berechnen lasse, seien Menschen bei der qualitativen Deutung unersetzbar. Anstrengungen, künstliche neuronale Netzwerke zu einer Allgemeinen KI weiterzuentwickeln, die Menschen ersetzen soll, weist Krüger dagegen zurück. In anthropologischer Hinsicht speisten sich die Grenzen der Utopien über eine Ersetzung der Menschen durch eine Superintelligenz aus ihren unterkomplexen Vorstellungen von menschlicher Intelligenz. In politischer Hinsicht macht Krüger die posthumanistischen Utopien als Herrschaftsideologien ökonomischer und militärischer Oligopole durchsichtig.

Aus den Perspektiven philosophischer Anthropologie, Kultur- und Technikphilosophie verorten Armin Grunwald, Oliver Müller und Jos de Mul die transhumanistischen Utopien in längeren geistesgeschichtlichen Traditionen. Armin Grunwald unterscheidet in seinem Aufsatz zwischen technischen Visionen und eschatologischen Erzählungen in der Tradition religiöser Erlösungslehren. Der Schritt in Technikeschatologien werde gemacht, wenn etwa Geschichten von der Erlösung von Sterben und Tod oder von der Herstellung ewiger Gerechtigkeit durch KI erzählt werden. Auch wenn mit KI und Robotik autonome technische Systeme entwickelt worden sind, die immer weitere Aspekte menschlichen Verhaltens und menschlicher Leistung ersetzen können und dabei oft effizienter sind als Menschen, fehlt derartigen Hoffnungen nach Grunwald die Erkenntnis- 
grundlage. Obgleich Hoffnungen auf eine Erlösung durch Technik unberechtigt seien, haben sie nach Grunwald jedoch einen nicht zu unterschätzenden Einfluss darauf, wie Mensch-Sein in der Gegenwart gedeutet und gelebt werde.

Oliver Müller setzt an den menschlichen Selbstverhältnissen an. Er reiht das posthumanistische Programm, die Evolution gezielt zu steuern, um Menschen biotechnologisch zu optimieren, in die geistesgeschichtliche Tradition menschlicher Selbstvervollkommnung und -optimierung ein. Innerhalb dieser Tradition markiert er eine Entwicklung, die vom Konversionsgebot über das Selbsterschaffungspostulat zum Selbstevolvierungsmandat verlaufe. Im kritischen Vergleich des Transhumanismus mit dem Existenzialismus zeigt Müller, dass die transhumanistische Agenda in letzter Konsequenz die Selbstabschaffung des Menschen zum Ziel hat. Dabei arbeitet er grundlegende Unterschiede zwischen dem transhumanistischen Pathos des Herstellens und dem existenzialistischen Pathos des Handelns, zwischen dem transhumanistischen Selbst-Design und dem existentialistischen Selbst-Entwurf sowie zwischen den unterschiedlichen Deutungen existenzieller Grenzsituationen heraus, denen der Transhumanismus entkommen wolle, während der Existenzialismus sie als Bedingungen der Möglichkeit von Selbstwerdung verstehe.

In Ergänzung zu Oliver Müller fokussiert Jos de Mul auf den Vergleich zwischen extra-, trans- und posthumanistischen Utopien und der Philosophischen Anthropologie von Helmuth Plessner. Zwar stimmten die Transhumanisten und Plessner darin überein, den Dualismus von Natur und Kultur zu unterlaufen; während Plessner die ,natürliche Künstlichkeit‘ jedoch als Grundgesetz der menschlichen Lebensform versteht, zielten die Transhumanismus darauf, die künstliche Durchdringung von Natur aktiv zu befördern, um auf diese Weise die menschliche in eine trans- oder postmenschliche Lebensform zu verwandeln. Aus einer an Plessner geschulten Perspektive tritt de Mul schließlich dafür ein, sich von den transhumanistischen Träumen einer Abschaffung unseres Mensch-Seins nicht begeistern zu lassen.

\subsection{Anthropologischer Ausblick: Leiblich-geistige Verschränkungen unter den Bedingungen disruptiver Technologien}

In Gestalt des Aufsatzes von Johannes Schick macht der Band an seinem Ende die Frage nach dem Verhältnis von leiblichem Leben und neuartigen Technologien auf. Schick versteht seinen Aufsatz als Beitrag der Aufklärung, um zur Mündigkeit im Verhältnis zu Digitaltechnologien zu befähigen. Dafür fokussiert er am leib- 
lichen Leben auf die Gesten als die leiblichen Akte, mit denen technische Objekte manipuliert werden. Schick tritt dafür ein, dass es in einem emphatischen Sinne keine ,natürlichen' Gesten gebe, sondern Gesten immer schon sozio-kulturell geformt seien. Bei ihrer Konstruktion werden in die technischen Objekte Weichenstellungen für die Gesten eingebaut, mit denen sie genutzt werden. Mittelbar werde dergestalt die Form der Gesellschaft durch die Art und Weise mitbestimmt, wie technische Objekte gestaltet, hergestellt und benutzt werden. Am Umgang mit smart phones erläutert Schick das Ineinandergreifen von geistig-mentalen und körper-leiblichen Operationen mit technischen Objekten: Gesten werden ausgeübt, um das smart phone logische Operationen ausführen zu lassen, die notwendig verborgen bleiben. Ein mündiges ,Denken mit den Händen‘ könne im digitalen Zeitalter gewonnen werden, indem - jenseits von einfachen Dualismen zwischen Körper und Geist - diese Durchdringung der eigenen Gesten zur Manipulation des smart phones durch die logischen Operationen reflektiert werde, die auf dessen Mikrochips ablaufen.

Der vorliegende Band bildet den dritten Band der Reihe „Grenzgänge. Studien in philosophischer Anthropologie“, die ich zusammen mit Reiner Anselm und Martin Heinze aufbaue. Die ersten beiden Bände haben sich das Gelingen des Sterbens (2019) bzw. das Gelingen der Geburt (2021) zum Gegenstand gemacht. Mit seiner Frage nach einem Gelingen der künstlichen Natürlichkeit führt der vorliegende Band ihre Auseinandersetzungen mit dem Gelingen des menschlichen Lebens an seinen Grenzen fort, das in den Debatten der zeitgenössischen Tugendethik unterbelichtet ist. Danken möchte ich an dieser Stelle allen Autorinnen und Autoren des Bandes für ihre klugen Aufsätze, Helena Hock für ihr sorgfältiges Korrekturlesen sowie Serena Pirrotta und Marcus Böhm vom de Gruyter Verlag für die reibungslose Zusammenarbeit.

\section{Literatur}

Arendt, Hannah (2012): Das Urteilen. Texte zu Kants politischer Philosophie, hg.v. Ronald Beiner, München/Zürich.

Christensen, Clayton M. (2011): The Innovator's Dilemma. Warum etablierte Unternehmen den Wettbewerb um bahnbrechende Innovationen verlieren, München.

Coenen, Christopher (2008): Konvergierende Technologien und Wissenschaften. Der Stand der Debatte und politischen Aktivitäten zu Converging Technologies, in: Büro für Technikfolgenabschätzung beim Deutschen Bundestag, Hintergrundpapier Nr. 16, https:// www.itas.kit.edu/pub/v/2008/coen08a.pdf., zuletzt abgerufen am 26.4.2021. 
Deutscher Ethikrat (2020): Stellungnahme: Robotik für gute Pflege, in: https://www.ethikrat. org/fileadmin/Publikationen/Stellungnahmen/deutsch/stellungnahme-robotik-fuer-gutepflege.pdf, zuletzt abgerufen am 26.4.2021.

Deutscher Ethikrat (2021): Öffentliche Anhörung: Künstliche Intelligenz und Mensch-Maschine-Schnittstellen, in: https://www.ethikrat.org/anhoerungen/kuenstlicheintelligenz-und-mensch-maschine-schnittstellen/, zuletzt abgerufen am 26.4.2021.

Jaeggi, Rahel (2014): Kritik von Lebensformen, Berlin.

Knell, Sebastian/Weber, Marcel (Hg. 2009): Länger leben? Philosophische und biowissenschaftliche Perspektiven, Frankfurt a. Main.

Leopoldina (2019): Stellungnahme: Fortpflanzungsmedizin in Deutschland - für eine zeitgemäße Gesetzgebung, in: https://www.leopoldina.org/uploads/tx_leopublication/ 2019_Stellungnahme_Fortpflanzungsmedizin_web_01.pdf, zuletzt abgerufen am 26.4.2021.

Max-Planck-Gesellschaft (2021a): Von Corona angesteckt, in: https://www.mpg.de/besuch-bei-viola-priesemann, zuletzt abgerufen am 26.4.2021.

Max-Planck-Gesellschaft (2021b): Communitas-Preis für Viola Priesemann, in: https://www.mpg.de/16444679/communitas-preis-fuer-viola-priesemann, zuletzt abgerufen am 26.4.2021.

Mitscherlich-Schönherr, Olivia/Anselm, Reiner (Hg. 2021): Gelingende Geburt. Interdisziplinäre Erkundungen in umstrittenen Terrains, Berlin. 